\title{
单点缺陷氧化石墨烯电子结构与光学特性的第一性原理研究
}

\author{
林启民 ${ }^{1}$, 崔建功 ${ }^{2}$, 颜 金金 ${ }^{1}$, 袁学光 ${ }^{1}$, 陈小瑜 ${ }^{1}$, 芦启超 ${ }^{1}$, \\ 罗彦涁 ${ }^{1}$, 黄 雪 ${ }^{3}$, 张 霞 ${ }^{1}$, 任晓敏 ${ }^{1}$
}

(1. 北京邮电大学 信息光子学与光通信国家重点实验室, 北京 $100876 ; 2$. 中北大学 电子测试技术重点实验室, 太原 030051; 3. 北京市计算中心，北京 100094)

摘 要: 本研究采用基于密度泛函理论的第一性原理方法, 在局域密度近似和广义梯度近似下, 研究了单点缺陷 下不同结构氧化石墨烯的电子结构和光学特性。研究结果表明: 文中四种构型的氧化石墨烯为力学稳定结构, 其 中包含不饱和氧原子的氧化石墨烯结构在水裂解及制氢中具有重要应用潜力。能带及分波态密度计算结果表明, 包含不饱和氧原子的构型为间接带隙半导体, 其余构型均为直接带隙半导体, 且掺杂类型和带隙值随结构不同而 改变。氧化石墨烯的光学吸收表现为各向异性, 且在垂直于平面方向上的吸收边蓝移到近紫外可见光区。包含 $\mathrm{sp}^{3}$ 杂化形式的结构光学吸收系数比包含 $\mathrm{sp}^{2}$ 杂化的结构高, 说明碳氧双键和悬挂键的存在对吸收光谱有重要影响。

关 键 词: 第一性原理; 氧化石墨烯; 光学性质; 能带; 吸收系数

中图分类号: O649 文献标识码: A

\section{First-principles Study on Electronic Structure and Optical Properties of Single Point Defect Graphene Oxide}

\author{
LIN Qimin ${ }^{1}$, CUI Jiangong ${ }^{2}$, YAN Xin ${ }^{1}$, YUAN Xueguang ${ }^{1}$, CHEN Xiaoyu ${ }^{1}$, LU Qichao ${ }^{1}$, \\ LUO Yanbin ${ }^{1}$, HUANG Xue ${ }^{3}$, ZHANG Xia $^{1}$, REN Xiaomin ${ }^{1}$
}

(1. State Key Laboratory of Information Photonics \& Optical Communications, Beijing University of Posts and Telecommunications, Beijing 100876, China; 2. State Key Laboratory of Dynamic Testing Technology, North University of China, Taiyuan 030051, China; 3. Beijing Computing Center, Beijing 100094, China)

\begin{abstract}
In this study, the electronic structure and optical properties of graphene oxide in different structures with single point defect are studied under local density of states approximation and generalized gradient approximation by first-principles calculations based on the density functional theory. The results show that four models are mechanically stable, among which the oxide graphene containing unsaturated oxygen atoms shows an important application potential in water cracking and catalysis. The calculated band structures and partial-wave density of states show that the model containing unsaturated oxygen atoms exhibits indirect band gap, while other models exhibit direct band gap, and the doping type and band gap values vary with different models. The absorption spectrum of graphene oxide is anisotropic, and the absorption edge moves to the near-UV and visible region in the direction perpendicular to the plane.
\end{abstract}

收稿日期：2019-11-20; 收到修改稿日期：2019-12-09

基金项目: 国家自然科学基金(61774021, 61911530133, 61935003); 信息光子学与光通信国家重点实验室(北京邮电大学)自 主研究课题(IPOC2019ZT07); 山西省青年科技研究基金(201801D221198); 山西省高等学校科技创新项目 (2019L0541); 中央高校基本科研业务费(2018XKJC05)

National Natural Science Foundation of China (61774021, 61911530133, 61935003); The Fund of State Key Laboratory of Information Photonics and Optical Communications (Beijing University of Posts and Telecommunications) (IPOC2019ZT07); Natural Science Foundation of Shanxi (201801D221198); STIP (2019L0541); Fundamental Research Business Expenses of Central Universities (2018XKJC05)

作者简介：林启民，男，博士研究生. E-mail: lqm@bupt.edu.cn LIN Qimin, male, PhD candidate. E-mail: 1qm@bupt.edu.cn

通讯作者: 袁学光, 讲师. E-mail: yuanxg@bupt.edu.cn; 张 霞, 教授. E-mail: xzhang@bupt.edu.cn YUAN Xueguang, lecturer. E-mail: yuanxg@bupt.edu.cn; ZHANG Xia, professor. E-mail: xzhang@bupt.edu.cn 
plane. The optical absorption coefficient containing $\mathrm{sp}^{3}$ hybrid is slightly higher than that containing $\mathrm{sp}^{2}$ hybrid, suggesting that the carbon-oxygen double bond and hanging bond have important influence on the absorption spectrum.

Key words: first-principles calculation; graphene oxide; optical property; band; absorbance

石墨烯因质量轻、机械强度高、导电性好和吸 收系数低等优点在近年受到广泛关注 ${ }^{[1-5]}$ 。石墨烯薄 膜不允许分子或离子通过, 而其氧化物即氧化石墨 烯则可实现半透膜的功能, 极大拓展了石墨烯的应 用 ${ }^{[6]}$ 。譬如, 氧化石墨烯因其良好的选择透过性可作 为微超级电容中的电解质实现电极分离 ${ }^{[7]}$; 基于氧 化石墨烯的湿度探测器具有循环稳定、成本低等优 势 ${ }^{[8]}$; 氧化石墨烯经过还原可作为电极、气敏探测

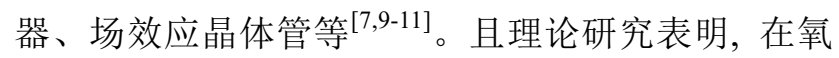
化石墨烯中掺杂 $\mathrm{Mg}$ 可以实现氢气的定向输运及存 储 ${ }^{[12]}$ 。可见, 氧化石墨烯具有极为广阔的应用前景, 开展相关理论和实验研究具有重要意义 ${ }^{[13-18]}$ 。

近年来, 已有大量关于氧化石墨烯的实验报 道。硝酸熏蒸石墨烯 ${ }^{[19]}$ 的 XPS 测试结果显示熏蒸前 后石墨烯均具有碳氧单键和双键。通过高猛酸钾和 双氧水等复杂工艺制备的氧化石墨烯 ${ }^{[20-21]}$ 具备环 氧、羟基等多种官能团。通过化学气象沉积合成同 位素标定石墨烯制备的氧化石墨烯 ${ }^{[22]}$ 包含以 $\mathrm{sp}^{2}$ 杂 化结合的羟基与环氧键以及碳氧双键等。实验结果 ${ }^{[23]}$ 还表明氧化石墨烯中存在碳氧单键、双键、环氧键 以及羧基等官能团, 且官能团的比例和光学性质随 温度变化而改变。上述实验结果表明, 不同工艺制 备的氧化石墨烯中碳氧键的形式及比例不同, 导致 其呈现不同的物理性质, 因此在理论上构建不同官 能团类型的氧化石墨烯并预测其物理特性是十分必 要的。目前, Yan 等 ${ }^{[24]}$ 已通过吸附方法初步构建出具 有羟基和环氧键的氧化石墨烯, 氧化石墨烯稳定性 和气敏相关氨气吸附氧化石墨烯的稳定性及其方向 性成功通过理论计算得出 ${ }^{[25-26]}$, 但更为全面的氧化 石墨烯模型及其详尽的物理性质仍有待深入研究。

本研究采用基于密度泛函理论的第一性原理计 算方法, 建立了单点缺陷下包含不同类型氧原子官 能团的氧化石墨烯模型, 并详细分析了不同模型的 电子结构和光学特性, 结果表明氧化位点的结构形 式对氧化石墨烯的能带结构、带隙宽度、半导体类 型和光学吸收系数等均有重要影响。

\section{1 计算方法与结构模型}

\section{1 计算方法}

采用基于密度泛函理论的第一性原理软件包
VSAP 进行计算 ${ }^{[24,27-28]}$ 。计算力学性质采用局域密 度泛函(LDA)的超软噟势进行计算, 平面波截断能 统一采用 $500 \mathrm{eV}$, 布里渊区空间的 $\mathrm{k}$ 点取样采用 Gamma 方法, 网格设为 $5 \times 5 \times 1$, 总能收玫标准优于 $1 \times 10^{-6} \mathrm{eV}$ 。原子结构模型驰豫、能带结构、态密度、 光学性质计算涉及的交换关联能采用广义梯度(GGA) 近似下的 Perdew-Burke-Ernzerhof(PBE)势, 采用缀 加平面波法 $(\mathrm{PAW})$, 总能收玫设为 $1 \times 10^{-4} \mathrm{eV}$, 单原 子作用力收敛标准设为 $-1 \times 10^{-3} \mathrm{eV} / \mathrm{nm}$ 。能带计算采 用倒空间高对称点线性模式, 两点间插入 10 个点。 为避免周期性的干扰, 所有计算过程中层间距设置 大于 $1.5 \mathrm{~nm}$ 。

\section{2 结构模型}

石墨烯为蜂窝状结构, 为简化计算, 本研究采 用正交坐标建模计算。计算以包含 16 个碳原子的 石墨烯结构为出发点，所有模型在此基础上构建: 除吸附羟基模型，其余模型均为缺失一个碳原子的 模型, 由 15 个碳原子和相应的氧原子以及氢原子 组成。

图 1 中(a)为基于石墨烯结构的单点吸附模型, (b) 为单点替位环氧模型, (c)为碳氧双键与环氧键共 存模型, (d)为包含不饱和氧原子模型。对结构(c)分 析可知单点缺陷氧原子并未达到饱和, 仍然可以吸 附氧, 因此构建双侧对称化吸附氧原子, 计算结果 列在表 1 中的 $\mathrm{d}_{1}$ 和 $\mathrm{d}_{2}$ 部分。结果显示两模型最终驰 豫形态一致, 键长数据基本一致, 据此确定其为同 一种模型结构, 即图 1 中的(d)。通过表 1 键长数据 结果可知这三个氧原子并不是以同样价态出现, 前 两种碳氧键为双键, 后一种为单键。图 1(d)包含的 碳氧单键出现了不饱和氧原子, 即存在氧的悬挂键, 使该结构化学性质活泼。通过对其进行氢原子钝化 以消除悬挂键, 构建的模型如图 1(e, f)所示。

\section{2 计算结果与讨论}

\section{1 结构属性及其稳定性}

图 1 中 (a d)、(e)和(f)三类结构的形成能按文献 [29]中相关公式计算,

$$
\begin{gathered}
\mathrm{C}_{n}+\mathrm{O}_{2}+\mathrm{H}_{2} \mathrm{O} \rightarrow \mathrm{C}_{n-1} \mathrm{O}_{x} \mathrm{H}_{y}+\mathrm{CO}_{2} \\
E_{\mathrm{f}}=E_{\mathrm{e}}-E_{\mathrm{b}}
\end{gathered}
$$



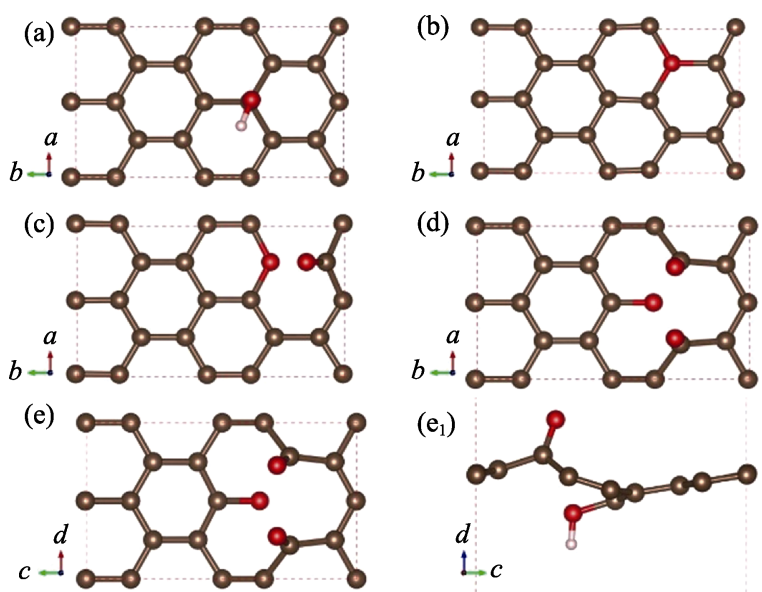

$\left(\mathrm{e}_{1}\right)$

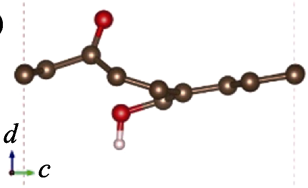

(f)
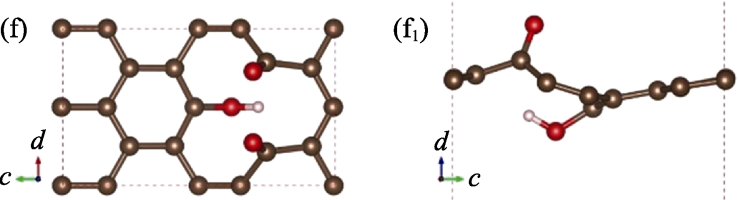

图 1 不同构型氧化石墨烯结构

Fig. 1 Different types of graphene oxide structures

(a) Graphene oxide adsorbed with hydroxyl; (b) Graphene oxide with single substitution epoxy bond; (c) Graphene oxide with carbon oxygen double bond and $\mathrm{sp}^{3}$ hybrid epoxy bond; (d) Graphene oxide with two carbon-oxygen double bonds and one carbon-oxygen single bond; (e, f) and $\left(e_{1}, f_{1}\right)$ Top and side views of the structure in (d) adsorbed with hydrogen on the upper and lower side of the suspended oxygen atom, respectively

表 1 不同泛函计算的不同结构中的键长 $(\mathbf{n m})$

Table 1 Bond length in different structures calculated by different pseudopotential functions (nm)

\begin{tabular}{ccccc}
\hline & $*$ & $\mathrm{O}_{1}-\mathrm{C}_{2}$ & $\mathrm{O}_{2}-\mathrm{C}_{6}$ & $\mathrm{O}_{3}-\mathrm{C}_{12}$ \\
\hline \multirow{6}{*}{ LDA } & $\mathrm{d}_{1}$ & 0.128 & 0.128 & 0.137 \\
& $\mathrm{~d}_{2}$ & 0.128 & 0.128 & 0.136 \\
& $\mathrm{e}$ & 0.124 & 0.124 & 0.138 \\
& $\mathrm{f}$ & 0.124 & 0.124 & 0.137 \\
\hline GGA & $\mathrm{d}$ & 0.124 & 0.124 & 0.133 \\
& $\mathrm{e}$ & 0.122 & 0.122 & 0.139 \\
& $\mathrm{f}$ & 0.122 & 0.122 & 0.138 \\
\hline
\end{tabular}

* (d) Graphene oxide with two carbon-oxygen double bonds and one carbon-oxygen single bond; $\left(\mathrm{d}_{1}, \mathrm{~d}_{2}\right)$ The precursors of (d); (e, f) Structure (d) adsorbed with hydrogen on the upper and lower side of the suspended oxygen atom, respectively

其中(1)式为合成示意式, 式中 $x, y$ 变化调节不同氧 化石墨烯的化学式。(2)式中 $E_{\mathrm{f}}$ 是形成能, $E_{\mathrm{b}} 、 E_{\mathrm{e}}$ 分 别为(1)式始末各成分的总能。相对应计算结果列于 表 2, 各结构形成能为负值, 说明可以存在。

图 1(a f) 六种模型的力学稳定性计算结果如表 3 所示。二维正交结构稳定性判定条件如下 ${ }^{[28]}$ :

$$
\begin{aligned}
C_{11} C_{22} & >C_{12}^{2}, \\
C_{66} & >0, \\
C_{11} & >0,
\end{aligned}
$$

式(3 5)中各项为相应基矢方向的弹性常数。

\section{表 2 各氧化石墨烯结构形成能 $(\mathrm{eV})$}

Table 2 Formation energy with different structures $(\mathrm{eV})$

\begin{tabular}{ccccccc}
\hline$*$ & (a) & (b) & (c) & (d) & (e) & (f) \\
\hline LDA & -12.7 & -6.1 & -6.5 & -13.1 & -19.6 & -18.6 \\
\hline
\end{tabular}

* (a) Graphene oxide adsorbed with hydroxyl; (b) Graphene oxide with single substitution epoxy bond; (c) Graphene oxide with carbon oxygen double bond and $\mathrm{sp}^{3}$ hybrid epoxy bond; (d) Graphene oxide with two carbon-oxygen double bonds and one carbon-oxygen single bond; (e) and (f) Structure (d) adsorbed with hydrogen on the upper and lower side of the suspended oxygen atom, respectively

\section{表 3 不同结构形式氧化石墨烯的弹性系数}

Table 3 Elastic coefficients of graphene oxide with different structures

\begin{tabular}{rrrrr}
\hline$*$ & $\mathrm{C}_{11}$ & \multicolumn{1}{c}{$\mathrm{C}_{22}$} & $\mathrm{C}_{12}$ & \multicolumn{1}{c}{$\mathrm{C}_{66}$} \\
\hline $\mathrm{a}$ & 1738.99 & 1618.45 & 282.75 & 1.03 \\
$\mathrm{~b}$ & 1789.57 & 1761.01 & 309.15 & 1.63 \\
$\mathrm{c}$ & 1607.47 & 958.73 & 163.94 & 0.99 \\
$\mathrm{~d}$ & 2015.11 & 1405.41 & 147.00 & 1.39 \\
$\mathrm{e}$ & 1968.65 & 825.43 & 148.48 & -11.90 \\
$\mathrm{f}$ & 1846.90 & 710.38 & 240.54 & -8.83 \\
\hline
\end{tabular}

* (a) Graphene oxide adsorbed with hydroxyl; (b) Graphene oxide with single substitution epoxy bond; (c) Graphene oxide with carbon oxygen double bond and $\mathrm{sp}^{3}$ hybrid epoxy bond; (d) Graphene oxide with two carbon-oxygen double bonds and one carbon-oxygen single bond; (e, f) Structure (d) adsorbed with hydrogen on the upper and lower side of the suspended oxygen atom, respectively

由以上 3 式结合表 2 中的数据, 可判定图 1 中 $(\mathrm{a} \sim \mathrm{c}) 、(\mathrm{~d})$ 结构均为稳定结构, 而图 1(e, f)两种结构 受力不稳定。因此, 包含不饱和氧原子的 (d) 结构可 稳定存在，而无悬挂键状态的图 1(e, f)结构不能稳 定存在。即在受到外力或扰动时, 氢原子可从无悬

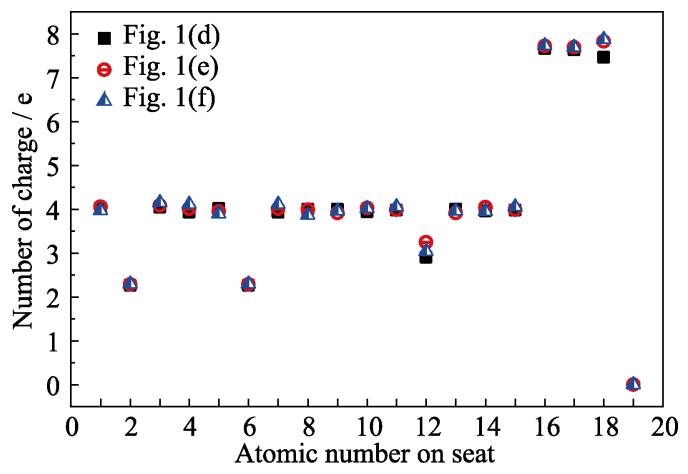

图 2 三种结构中不同原子的电荷数

Fig. 2 The charge number of three kinds of different atoms in three structures showing in Fig. 1(d), (e) and (f)

(d) Graphene oxide with two carbon-oxygen double bonds and one carbon-oxygen single bond; (e, f) Structure (d) adsorbed with hydrogen on the upper and lower side of the suspended oxygen atom, respectively 
挂键状态的氧化石墨烯表面解吸附, 形成包含不饱 和氧原子的氧化石墨烯。利用该过程可以进行裂解 水及其相关结构而实现析氢或制备氢源: 在有悬挂 键状态时((d)结构)可吸附氢原子形成(e)或(f)结构, 受力或外界扰动后释放氢原子后又回到( $\mathrm{d}$ )结构, 形 成一个持续不间断的制氢过程。

为计算各模型的电子结构和光学性质, 采用 GGA 方法对上述结果进行重新计算。表 1 列出了部 分键长, 同一结构略有变化是由于采用的泛函不同 引起。前两种键长为碳氧双键, 后一种键长为碳氧 单键。Bader 电荷分析计算结果如图 2 所示。图中 16-18 号原子为表 1 中编号为 1-3 的氧原子, 19 号为 氢原子, 其余均为碳原子。从图 2 中可以看出, 由于 图 2 中 $(\mathrm{d})$ 结构不存在 19 号氢原子, $2 、 6$ 号碳原子失 去约 2 个电子, 12 号碳原子失去约 1 个电子。结合
表 1 中的键长数据, 表明 2、6 号碳原子与 $1 、 2$ 号 氧原子连接为碳氧双键, 12 号碳原子与 3 号氧原子 相连为碳氧单键。图 2 中 $(\mathrm{d})$ 结构的 18 号原子即 3 号氧原子相对图 2 中 $(\mathrm{e}, \mathrm{f})$ 电荷数较少, 为不饱和状态 (即含有悬挂键), 与键长分析结果一致。

综上所述, 图 1 中 (a $\sim \mathrm{d})$ 四种结构稳定, 图 1 中(b) 结构各原子在同一平面为 $\mathrm{sp}^{2}$ 杂化, 其余结构缺陷 附近碳原子与其余碳原子不再共面为 $\mathrm{sp}^{3}$ 杂化; 图 1 中 $(\mathrm{e}, \mathrm{f})$ 两种结构实现对图 1 中 $(\mathrm{d})$ 结构中氧原子悬挂 键的针化。图 1 中 (c) 结构具有一个碳氧双键, 图 1 中 $(\mathrm{d})$ 结构具有两碳氧双键和一个具有氧原子悬挂 键的碳氧单键。(d)结构用于析氢反应。

\section{2 电子结构}

为分析以上模型的电子结构性质, 计算得出能 带结构和分波态密度(Density of states, DOS)如图 3
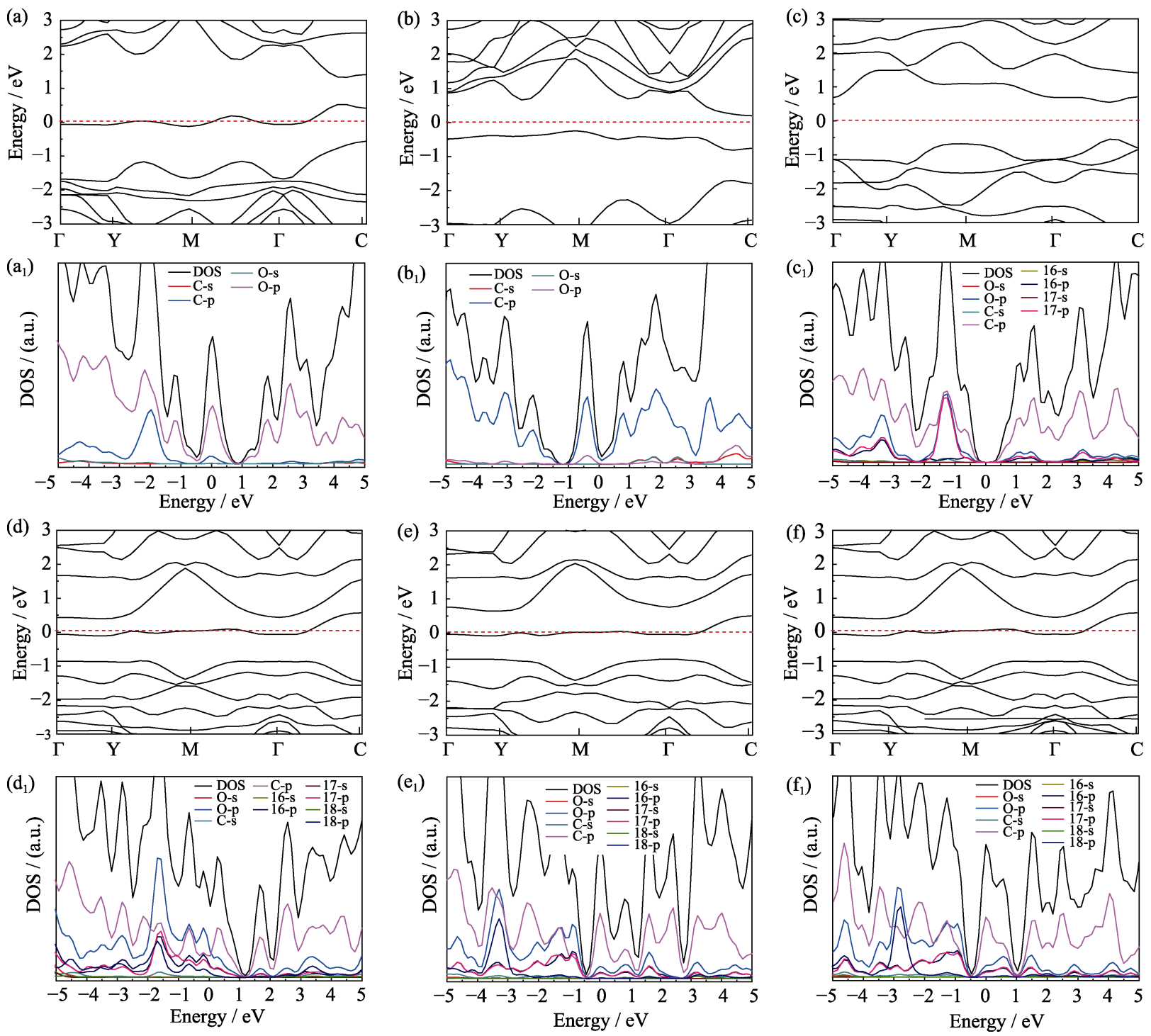

图 3 各结构能带结构及态密度图

Fig. 3 Band structures and density of states (DOS) of different structure models

(a) Graphene oxide adsorbed with hydroxyl; (b) Graphene oxide with single substitution epoxy bond; (c) Graphene oxide with carbon oxygen double bond and $\mathrm{sp}^{3}$ hybrid epoxy bond; (d) Graphene oxide with two carbon-oxygen double bonds and one carbon-oxygen single bond; (e,f) Structure (d) adsorbed with hydrogen on the upper and lower side of the suspended oxygen atom, respectively 
所示。图 3(a)为单点吸附模型的能带结构, 可以看 出该结构为直接带隙, 带隙值为 $0.79 \mathrm{eV}$, 结合其态 密度图(图 3( $\left.\left.\mathrm{a}_{1}\right)\right)$ 分析显示费米能级进入价带, 为 $\mathrm{p}$ 型半导体。图 3(b)为单点替位环氧模型的能带结构, 该结构为直接带隙, 带隙值为 $0.91 \mathrm{eV}$, 且费米能级 进入导带，属于 $\mathrm{n}$ 型半导体。图 3(c) 为碳氧双键与 环氧键共存模型的能带结构图, 显示该结构为直接 带隙，带隙值为 $1.1 \mathrm{eV}$, 属于本征半导体。图 3(d) 为包含不饱和氧模型的能带结构图, 结合其 DOS 分 析显示为间接带隙, 费米能级在价带中, 为 $\mathrm{p}$ 型半 导体。图 3(e, f) 分别为图 1 中 $(\mathrm{e}, \mathrm{f})$ 结构的能带结构, 均为直接带隙, 带隙值分别为 0.72 和 $0.82 \mathrm{eV}$, 且费 米面均位于导带, 属 $\mathrm{n}$ 型半导体。由以上分析可知, 石墨烯氧化位点形式不同, 其半导体类型和带隙值 亦不同。

图 3( $\left.a_{1}, b_{1}\right)$ 显示结构 $(a, b)$ 碳氧相互作用主要来 自于碳和氧的 $\mathrm{p}$ 轨道电子相互作用。图 3( $\left.\mathrm{c}_{1}\right)$ 显示结 构(c)的碳氧相互作用同样来自于 $\mathrm{p}$ 轨道电子, 但进 一步的氧轨道分析显示其相互作用主要来源于形成 碳氧双键的 17 号原子的 $\mathrm{p}$ 轨道, 而碳氧单键中的氧
原子贡献基本为零。显然, 在此结构中碳氧双键对 带隙的影响强于碳氧单键。图 3 $\left(\mathrm{d}_{1}\right)$ 说明 $(\mathrm{d})$ 结构中氧 原子的 $\mathrm{p}$ 轨道电子对带隙的贡献明显增大, 且主要 贡献来源于两个碳氧双键中的 16、17 号原子。结合 bader 电荷分析表明, 这种作用主要来源于两个碳 氧双键, 而处于悬挂键位置的 18 号氧原子对带隙也 有贡献但弱于碳氧双键。图 3( $\left.\mathrm{e}_{1}, \mathrm{f}_{1}\right)$ 说明, 对于不存 在氧原子悬挂键的结构, 被钝化的 3 号氧原子的 $\mathrm{p}$ 轨道在费米能级附近为零, 因此对带隙无影响。以 上分析表明，对于 (d)结构氧化石墨烯参与的水裂解 或析氢过程来说, 氧原子的 $\mathrm{p}$ 轨道悬挂键起着重要 作用。

\section{3 光学性质}

根据光吸收谱公式 ${ }^{[4]}$

$$
I(\omega)=\sqrt{2} \omega\left[\sqrt{\varepsilon_{1}^{2}(\omega)+\varepsilon_{2}^{2}(\omega)}-\varepsilon_{1}(\omega)\right]^{\frac{1}{2}}
$$

计算得到文中各结构的光学吸收谱如图 4 所示。(6) 式中 $\varepsilon_{1} 、 \varepsilon_{2}$ 分别是介电函数的实部和虚部, $\omega$ 是光子 的频率。
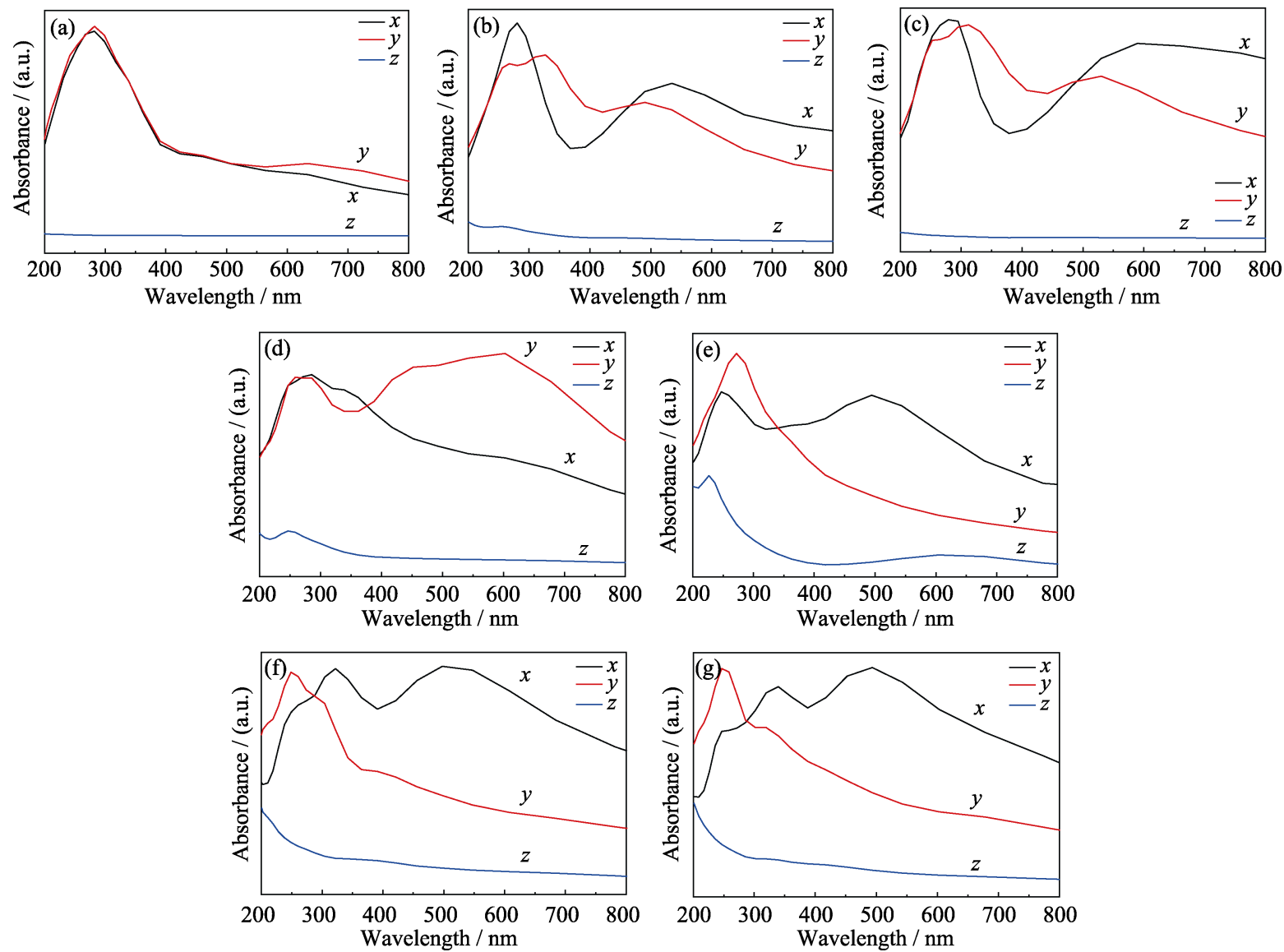

图 4 各结构光学吸收系数

Fig. 4 Absorption coefficient of different structure models in which (b-g) the absorption coefficient of graphene and structures in Fig. 1(a-f) (a) Graphene; (b) Graphene oxide adsorbed with hydroxyl; (c) Graphene oxide with single substitution epoxy bond; (d) Graphene oxide with carbon oxygen double bond and $\mathrm{sp}^{3}$ hybrid epoxy bond; (e) Graphene oxide with two carbon-oxygen double bonds and one carbon-oxygen single bond; (f, g) Structure (d) adsorbed with hydrogen on the upper and lower side of the suspended oxygen atom, respectively 
图 4(a)为石墨烯在三个坐标轴方向的吸收光谱, 可以看出在 $x 、 y$ 方向基本一致，表现为各向同性， 在 $\mathrm{z}$ 轴方向吸收系数较低且变化趋势平缓。从图 4(b g) 可以看出不同的单点缺陷氧化石墨烯结构均 表现出光学吸收的各向异性, 且在可见光区吸收系 数相对石墨烯增强; $z$ 轴方向在紫外区的吸收系数增 加明显, 表明带隙值有所增加, 与能带结构的计算 结果一致。其中, 图 4(c)为图 1 中(b)结构在 $z$ 轴方 向的吸收图谱, 明显相对其它氧化石墨烯结构的吸 收较低, 可能是为 $\mathrm{sp}^{2}$ 杂化所致。图 4(d,e)为结构(c) 和(d)的光学吸收谱, 它们在 $z$ 方向的吸收均明显强 于图 4(b)结构。结合前文中的键长和电荷密度分析, 可知是由于碳氧双键在其中起关键作用, 且图 4(d) 结构的吸收系数还有碳氧单键中氧原子的悬挂键贡 献。图 4(f, g) 为氢原子钝化后的结构的光学吸收谱, 它们基本保持一致, 微小差异来源于结构的细微差 别。其吸收系数较图 4(c)结构高是由于碳氧双键数 量增多。

综上可知, 石墨烯氧化后表现为光学吸收的各 向异性, 吸收峰蓝移且在紫外一可见光区吸收增强, 提高了在可见光区的光催化活性。包含 $\mathrm{sp}^{3}$ 杂化形 式的结构吸收谱强于包含 $\mathrm{sp}^{2}$ 杂化形式的结构, 且 碳氧双键和悬挂键对光谱吸收起到重要作用。

\section{3 结论}

本研究采用基于密度泛函理论的第一性原理方 法，在局域密度近似和广义梯度近似下研究了单点 缺陷下不同结构氧化石墨烯的电子结构和光学特 性。研究结果表明文中的四种结构的氧化石墨烯均 为力学稳定结构, 其中包含不饱和氧原子的结构中 存在的单氧悬挂键为实现催化制氢反应提供了可 能。氧化石墨烯表现出半导体特性, 其中包含不饱 和氧原子的结构表现为间接带隙半导体, 其余结构 为直接带隙半导体, 且半导体的掺杂类型和带隙值 随结构的不同而不同。各种结构氧化石墨烯的光学 吸收谱均表现为各向异性, 且在 $\mathrm{z}$ 轴方向氧化石墨 烯的吸收系数较石墨烯均有不同程度的提高。包含 且 $\mathrm{sp}^{3}$ 杂化形式的结构较包含 $\mathrm{sp}^{2}$ 杂化形式的结构具 有更高的吸收系数, 且碳氧双键和悬挂键对光谱吸 收起到重要作用。

\section{参考文献:}

[1] YANG K, SHUAI X R, YANG H C, et al. Electrochemical performance of activated graphene powder supercapacitors using a room temperature ionic ILiquid electrolyte. Acta Phys. -Chim. Sin., 2019, 35(7): 755-765.

[2] PERES N M R. The Transport properties of graphene. J. Phys. Condens. Matter., 2009, 21(32): 323201-323210

[3] CHEN D M. Variation of graphene Raman G peak splitting with strain. Acta. Phys.. Sin., 2010, 59(9): 6399-6404.
[4] WANG Y F, LI X W. First-principle calculation on electronic structures and optical properties of hybrid graphene and BiOI nanosheets. Acta. Phys. Sin., 2018, 67(11): 168-175.

[5] WANG J J, WANG F, YUAN P F, et al. First-principles study of nanoscale friction between graphenes. Acta Phys. Sin., 2012, 61(10): $337-343$

[6] JOSHI R K, ALWARAPPAN S, YOSHIMURA M, et al. Graphene oxide: the new membrane material. Applied Materials Today, 2015, 1: 1-12

[7] GAO W, SINGH N, SONG L, et al. Direct laser writing of microsupercapacitors on hydrated graphite oxide films. Nature Nanotechnology, 6: 496-500.

[8] WANG G X, PEI Z B, YE C H, et al. Inkjet-printing and performance investigation of self-powered flexible graphene oxide humidity sensors. Journal of Inorganic Materials, 2019, 34(1): 114-120.

[9] HUANG J R, WANG L Y, SHI C C, et al. Selective detection of picric acid using functionalized reduced graphene oxide sensor device. Sensors \& Actuators B Chemical, 196: 567-573.

[10] LI C, CAI L, LI W W, et al. Adsorption of $\mathrm{NO}_{2}$ by hydrazine hydrate-reduced graphene oxide. Acta Phys. Sin., 2019, 68(11): 257-262.

[11] PENG P, LIU H T, WU B, et al. Nitrogen doped graphene with a p-type field-effect and its fine modulation. Acta Phys. Chim. Sin., 2019, 35(11): 1282-1290.

[12] CHU C, ZHANG J, BEI Z, et al. Hydrogen adsorption of Mgdoped Graphene oxide: afirst-principles study. Journal of Physical Chemistry C, 2013, 117: 4337-4344.

[13] ROGERS G W, LIU J Z. High-performance graphene oxide electromechanical actuators. Journal of the American Chemical Society, 2012, 134: 1250-1255.

[14] ZHU Y, MURALI S, CAI W, et al. Graphene and graphene oxide: synthesis, properties, and applications. Cheminform, 2010, 22: 3906-3924.

[15] KIM S, ZHOU S, HU, Y, et al. Room-temperature metastability of multilayer graphene oxide films. Nature Materials, 2012, 11(6): 544-549.

[16] ZHAO H, ZHOU L, WEI D, et al. Effects of external electric field on hydrogen storage performance of Li-decorated graphene oxide. Chemical Journal of Chinese Universities, 37(1): 100-107.

[17] LOH K P, BAO Q, EDA G, et al. Graphene oxide as a chemically tunable platform for optical applications. Nature Chemistry, 2: $1015-1024$.

[18] ZHANG Q, ZHANG H, CHENG X L. Highly stable two-dimensional graphene oxide: electronic properties of its periodic structure and optical properties of its nanostructures. Chinese Physics B, 27(2): $027301-1-7$.

[19] BAE S, KIM H, LEE Y, et al. Roll-to-roll production of 30-inch graphene films for transparent electrodes. Nat. Nanotechnol, 2010, 5: $574-578$.

[20] COMPTON, O C, NGUYEN, S B T. Graphene oxide, highly reduced graphene oxide, and graphene: versatile building blocks for carbon-based materials. Small, 2010, 6: 711-723.

[21] HONG F, ZHOU L Q, HUANG Y, et al. Synthesis and characterization of graphene by improved hummers method. Chemistry \& Bioengineering, 2012, 29: 31-33.

[22] CAI W W, PINER R D, STADERMANN F J, et al. Synthesis and solid-state NMR structural characterization of 13c-labeled graphite oxide. Science, 2008, 321: 1815-1817.

[23] MO J W, QIU Y W, YI R B, et al. Temperature-dependent properties of metastable graphene oxide. Acta Phys. Sin., 2019, 68(15): 284-292.

[24] YAN J A, XIAN L, CHOU M Y. Structural and electronic properties of oxidized graphene. Phys. Rev. Lett., 2009, 103: 086802-1-4.

[25] WANG L, SUN Y Y, LEE K, et al. Stability of graphene oxide phases from firstp calculations. Physical Review B 2010, 82: 161406-1-4.

[26] PENG Y, LI J. Ammonia adsorption on graphene and graphene oxide: a first-principles study. Frontiers of Environmental Science \& Engineering, 2013, 7: 403-411.

[27] ZHANG Y, SHI Y M, BAO Y Z, et al. Effect of surface passivation on the electronic properties of GaAs nanowire: A first-principle study. Acta Phys. Sin., 2017, 66(19): 295-301.

[28] YI W C, HU T, SU T, et al. A CNH monolayer: a direct gap 2d semiconductor with anisotropic electronic and optical properties. Journal of Materials Chemistry C, 2017, 5: 8498-8503.

[29] LIN Q M, ZHANG X, LU Q C, et al. First-principles study on structural stability of graphene oxide and catalytic activity of nitric acid. Acta Phys. Sin., 2019, 68(24): 247302-1-6. 